\title{
BIOMECHANICAL CHANGES IN WOMEN WITH CHRONIC PELVIC PAIN: A CASE-CONTROL STUDY
}

original paper

() Wroclaw University of Health and Sport Sciences

DOI: https://doi.org/10.5114/hm.2021.104185

\section{ROVAN M. ELBESH ${ }^{1}$, HAMADA A. HAMADA ${ }^{2}$, MOHAMED A. ELANEN ${ }^{3}$, AMEL M. YOUSEF ${ }^{4}$, TAMER MOHAMED SHOUSHA ${ }^{5,6}$, RANIA REDA MOHAMED $^{7}$}

\author{
${ }^{1}$ Department of Physical Therapy for Women's Health, Faculty of Physical Therapy, Misr University for Science \\ and Technology, Giza, Egypt \\ ${ }^{2}$ Department of Biomechanics, Faculty of Physical Therapy, Cairo University, Cairo, Egypt \\ ${ }^{3}$ Om El-Masryeen Hospital, Cairo, Egypt \\ ${ }^{4}$ Department of Physical Therapy for Women's Health, Faculty of Physical Therapy, Cairo University, Cairo, Egypt \\ ${ }^{5}$ Physiotherapy Department, University of Sharjah, Sharjah, United Arab Emirates \\ ${ }^{6}$ Department of Physical Therapy for Musculoskeletal Disorders and Its Surgery, Faculty of Physical Therapy, \\ Cairo University, Egypt \\ ${ }^{7}$ Department of Basic Science, Faculty of Physical Therapy, Cairo University, Cairo, Egypt
}

\section{ABSTRACT}

Purpose. Chronic pelvic pain can influence women's quality of life and activity levels; it can lead to several adverse side effects, such as changes in posture and biomechanical alignment. The purpose of this study was to determine the biomechanical changes that occur in women experiencing chronic pelvic pain, either cyclic or noncyclic.

Methods. A case-control study was conducted among 60 females from the Gynecology Physiotherapy Clinic of El-Hosary Women Health Care in 6th of October city. The participants were assigned into 3 groups: cyclic chronic pelvic pain group (A), noncyclic chronic pelvic pain group (B), and normal women group (C). An inclinometer was used to assess biomechanical changes in the angles of the cervical, thoracic, and lumbar region; furthermore, a pelvic inclinometer served to measure the pelvic inclination angle.

Results. Statistical analysis revealed that there was a significant increase in biomechanical variables at the lumbar, thoracic, and cervical angles in group B compared with groups A and C and in group A compared with group C. Moreover, there was a significant increase in right and left pelvic inclination angles in groups A and B compared with group C, and no significant difference was observed between groups A and B.

Conclusions. Biomechanical alignment changes are seen more frequently in women with noncyclic chronic pelvic pain and cyclic chronic pelvic pain than in normal women.

Key words: biomechanical alignment, spinal alignment, chronic pelvic pain, cyclic pelvic pain, noncyclic pelvic pain

\section{Introduction}

Chronic pelvic pain (CPP) is persistent pain in the area below the belly button and between hips lasting for 3-6 months and of sufficient severity [1]. It is usually detrimental to a woman's health and requires a comprehensive medical evaluation and management $[2,3]$. CPP is classified into 2 types: cyclic CPP and noncyclic CPP. The noncyclic CPP occurs not in accordance with menstruation and is represented in the bottom abdomen or pelvis as persistent or chronic pain [3]. It has also an economic burden, being the cause of about $10-25 \%$ of all gynaecological consultations [4]. Cyclic CPP is traditionally considered a CPP type following the menstrual cycle, and it is characteristic of fertile age women [3]. CPP can involve a variety of physical causes, including pregnancy, as well as urinary, gynaecological, neurological, and musculoskeletal conditions [3]. Biomechanical, musculoskeletal, and postural changes are frequent in women with CPP

Correspondence address: Hamada A. Hamada, Faculty of Physical Therapy, Cairo University, 1 Gamaa Street, Giza, Egypt, e-mail: Hamada.Ahmed@pt.cu.edu.eg

Received: February 7, 2020

Accepted for publication: January 11, 2021

Citation: Elbesh RM, Hamada HA, Elanen MA, Yousef AM, Shousha TM, Mohamed RR. Biomechanical changes in women with chronic pelvic pain: a case-control study. Hum Mov. 2022;23(1):37-43; doi: https://doi.org/10.5114/hm.2021.104185. 
$[5,6]$, and extended impairments in biomechanical alignment might contribute significantly to pain maintenance [6]. Kim et al. [7] supposed that the pelvis and spine alignments caused structural and functional changes of the body; including the uterus, this could in turn increase cyclic CPP. However, few studies have determined the relationship between cyclic CPP and the pelvic biomechanics [5-7]. Furthermore, not so many studies have compared noncyclic and cyclic CPP. Therefore, the current study aims to determine the biomechanical changes that may occur in women with noncyclic and cyclic CPP to find out its effect on the configuration of the spine and pelvis.

\section{Material and methods}

\section{Participants}

From among 100 women eligible for the study from the Gynecology Physiotherapy Clinic of El-Hosary Women Health Care in $6^{\text {th }}$ of October city, only 60 participated in the research (Figure 1) and 40 were excluded. Patients were excluded from the study if they had an abnormal menstrual cycle (bleeding, amenorrhoea, irregularity, etc.), vaginal discharge, pelvic organ prolapse, uterine or ovarian mass, endometriosis, an intrauterine device applied, bloating in the abdomen, urinary tract infection that might have increased pelvic pain. Women who were pregnant or in the lactation phase and who received hormonal treatment were also excluded because that affects pelvic ligaments laxity and may increase pain. Patients with previous caesarean section or previous gynaecological or spinal sur- geries were excluded to avoid pain caused by adhesions. Patients with spinal deformities, leg length discrepancy, rectus diastasis, orthopaedic or neurological problems that affected the pelvis mechanics were also excluded. Moreover, we excluded women with cancer, diabetes mellitus, cardiovascular diseases, and psychological problems affecting general mental and physical status.

The participants' age ranged from 20 to 30 years, and their body mass index equalled $18-25 \mathrm{~kg} / \mathrm{m}^{2}$. Overall, 44 out of the 60 women selected in accordance with the inclusion criteria experienced persistent CPP of moderate intensity as evaluated with the visual analogue scale. The participants were asked to report at least 1 area painful within at least 6 months before the study, out of the following: lower back, sacroiliac joint, symphysis pubic joint, perineum, anterior or lower abdominal wall. The individuals were divided into 2 study groups: group A included 24 women with cyclic CPP (pain related to menstruation), while group B involved 20 women with noncyclic CPP. The remaining 16 normal women constituted a control group (C).

\section{Design and procedures}

This observational case-control study was conducted between November 2018 and May 2019.

All assessments of the 3 groups (A, B, and C) were performed on the first day after the menstrual cycle. Lumbar, thoracic, and cervical angles were measured with an inclinometer and were chosen as study parameters. We applied a rounded plastic inclinometer that

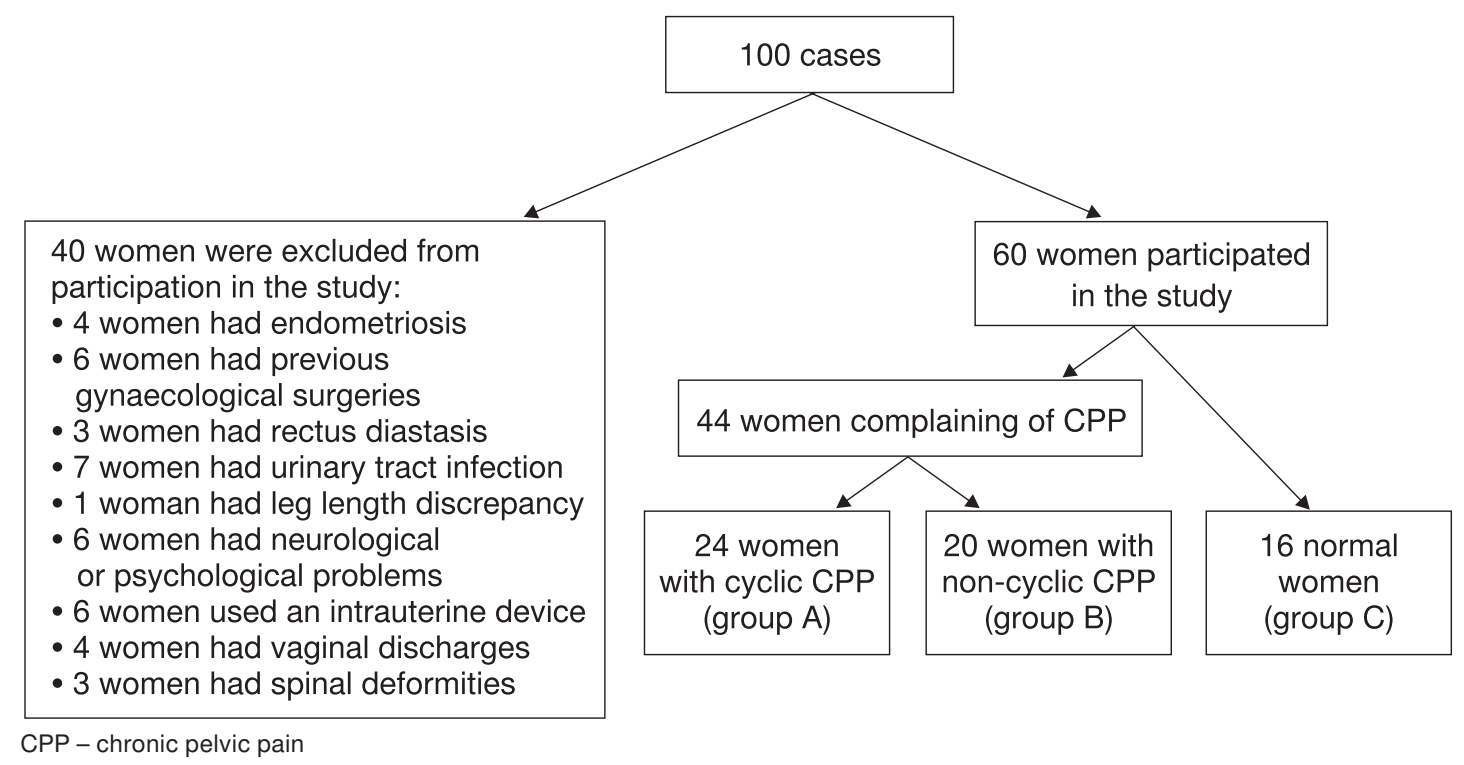

Figure 1. Flow diagram of the study 
had a $360^{\circ}$ rotating dial with a fluid indicator (NoLeak PT inclinometer for range of motion measurements by Sense Aid, White Plains, USA); pelvic angle was measured with a PALM inclinometer. The PALM inclinometer is an accurate tool for evaluating skeletal alignment in a clinical setting in both healthy and patient populations. It measures the tilt angle and space between any 2 marked points of the body. It can give the height discrepancy between 2 landmarks palpated. A calliper inclinometer instrument (Performance Attainment Associates, US Patent 5327907) consists of a bubble inclinometer and 2 calliper arms. The inclinometer is a semi-circular arc with $1^{\circ}$ gradation ranging from $0^{\circ}$ to $30^{\circ}$ on either side of the midline. The calliper arm tips are designed for direct palpation of the bony landmarks. The distance between the calliper arms can be measured via a mounted protractor (calliper dial) with 2-mm gradation and a range of $0-43 \mathrm{~cm}$. There was a fixed point in the wall, and each woman was instructed to look at it during the assessment [8]. Each angle was measured 3 times, and the mean was calculated.

\section{Outcome measures}

\section{Pelvic tilt angle}

The physiotherapist stood next to the female and noticed the location of the anterior superior iliac spine (ASIS) and the posterior superior iliac spine (PSIS). Thus, the therapist placed the PALM inclinometer at ASIS with one end arm and at PSIS with the other end arm. The angle of pelvis inclination was the angle between the horizontal line and the line that crossed ASIS and PSIS, calculated by the PALM inclinometer bubble level [9]. The previous measurement was performed on both sides of the pelvis. The accuracy and validity of this method exhibit high intra-tester reliability (intraclass correlation coefficient $[$ ICC $]=0.87$ ) [9]. The mean pelvic tilt angle value was $13 \pm 6^{\circ}[8]$.

\section{Cervical angle}

The physiotherapist stood behind the woman seated on a stool and identified the base of the $\mathrm{C}_{2}$ and $\mathrm{C}_{7}$ to measure cervical lordosis. Then the therapist put the inclinometer over the $C_{2}$ spinous process, with the 2 feet end of the inclinometer base placed in the interspinous spaces above and below the $\mathrm{C}_{2}$. The inclinometer was set to zero in the $\mathrm{C}_{2}$ position and moved over the $\mathrm{C}_{7}$ spinous process. The accuracy and validity of this method exhibit high intra-tester reliability
$(\mathrm{ICC}=0.84)$. The normal range for cervical lordosis is $31-40^{\circ}$ [10].

\section{Lumbar angle}

Before the examination, the physiotherapist identified the spinous processes of the $\mathrm{T}_{12}$ and $\mathrm{L}_{5}$, by applying a skin marker on the spinous processes of the $\mathrm{T}_{12}$ and $\mathrm{L}_{5}$. The lumbar angle was measured by the inclinometer dial, which was set to $0^{\circ}$ at the wall. Then, the physiotherapist put the inclinometer into the inter-spinal spaces between $\mathrm{T}_{12}$ and $\mathrm{L}_{5}$, thus obtaining the lumbar angle. Neutral values of the lumbar angle are $20-40^{\circ}$ [11]. The baseline inclinometer was used with high intra-rater reliability $($ ICC $=0.92)$ of the bubble inclinometer [12].

\section{Thoracic angle}

The physiotherapist stood behind the participant, and 2 skin markers were placed on the skin over the $\mathrm{C}_{7}$ and $\mathrm{T}_{12}$ spinous processes. To identify the position of the $\mathrm{C}_{7}$ spinous process by palpation, the therapist asked the patient to make an ahead extension and put their finger on both spinous processes of $\mathrm{C}_{6}$ and $\mathrm{C}_{7}$. The spinous process of $\mathrm{C}_{7}$ should not have moved, while the $\mathrm{C}_{6}$ spinous process moved anteriorly and then the thoracic spinous processes was identified by palpation of the spinous processes to $\mathrm{T}_{12}$. Using a standard clinical procedure, as outlined by Clarkson [13], the therapist placed the inclinometer on the skin markers at the $C_{7}$ spinous process to measure the cervical inclination angle. Then, this procedure was repeated for the lower thoracic spine to measure the thoracic inclination, with the caudal end of the inclinometer placed on the skin markers of $\mathrm{T}_{12}$. The thoracic kyphotic angle was measured by taking the difference between the 2 previous measurements from the inclinometer readings. The thoracic kyphosis curvature in the sagittal plane lies between the $T_{1}$ and $T_{12}$ vertebral bodies. The normal thoracic kyphotic angle ranged from $20^{\circ}$ to $45^{\circ}$. Hyperkyphosis is the kyphotic angle $>50^{\circ}[14]$.

\section{Statistical analysis}

The Statistical Package for the Social Sciences (SPSS) version 23 was utilized for statistical analysis. The recorded data were tested for normality and the presence of any extreme score. Such measures were considered before any parametric calculations regarding significant differences or relationships. The initial descrip- 
R.M. Elbesh et al., Biomechanical changes in chronic pelvic pain

tive statistics revealed a normal distribution of the parameters recorded and did not seem to violate the parametric assumption of the measured variables. Additionally, Box's test for homogeneity of covariance showed no significant differences, with $p$ values exceeding 0.05. The Shapiro-Wilk test revealed that all measured dependent variables were normally distributed. Consequently, parametric testing was applied, particularly the one way between-subject MANOVA, to detect the significance of variance of the tested variables for different groups. Also, the one way between-subject ANOVA test for demographic data was used. Significance was considered at $p<0.05$.

\section{Ethical approval}

The research related to human use has complied with all the relevant national regulations and institutional policies, has followed the tenets of the Declaration of Helsinki, and has been approved by the Institutional Review Board at the Faculty of Physical Therapy, Cairo University (NP.T.REC/012/001887, clinical trial No. NCT03740932).

\section{Informed consent}

Informed consent has been obtained from all individuals included in this study.

\section{Results}

Table 1 shows the participant characteristics of groups $\mathrm{A}, \mathrm{B}$, and $\mathrm{C}$. There was no significant difference in the mean age, weight, height, or body mass index between the 3 groups $(p>0.05)$.

One way between-subject MANOVA for the outcome measures indicated statistically significant effects for the group $\left(F=99.936, p=0.001\right.$, partial $\eta^{2}=$ 0.904). Comparing the results among the 3 tested groups, a significant increase was revealed $(p<0.05)$ (very large effect size) in the mean values of thoracic angle, lumbar angle, and cervical angle in group B compared with groups A and C. Also, there was a significant increase (large effect size) $(p<0.05)$ in these angles in group A compared with group C. Moreover, a significant increase was detected $(p<0.05)$ in the right and left pelvic inclination angles in groups $\mathrm{A}$ and B (large effect size) compared with group C, and there was no significant difference $(p>0.05)$ between groups A and B (small effect size), as shown in Table 2.

\section{Discussion}

We found significant differences in biomechanical alignment changes in women with CPP. The results of the current study revealed that there was a significant difference in biomechanical variables (lumbar, cervical lordosis, right pelvic angle, left pelvic angle, and thoracic kyphosis angles) between the 3 groups. This significant difference favoured the noncyclic CPP group (B) rather than the cyclic CPP group (A) compared with the control group (C), and no significant difference was observed between groups $\mathrm{A}$ and $\mathrm{B}$ in the right or left pelvic angle.

Table 1. Participant characteristics

\begin{tabular}{lccrl}
\hline \multirow{2}{*}{ Characteristics } & Group A & Group B & Group C & \multirow{2}{*}{$p$} \\
\cline { 2 - 4 } & Mean $\pm S D$ & Mean $\pm S D$ & Mean $\pm S D$ & \\
\hline Age (years) & $23.25 \pm 0.78$ & $22.87 \pm 0.67$ & $23.18 \pm 0.65$ & 0.18 \\
Weight $(\mathrm{kg})$ & $55.15 \pm 3.08$ & $54.83 \pm 1.9$ & $54.5 \pm 2.12$ & 0.72 \\
Height $(\mathrm{cm})$ & $162.5 \pm 7.68$ & $164.08 \pm 4.93$ & $162.25 \pm 3.53$ & 0.53 \\
Body mass index $\left(\mathrm{kg} / \mathrm{m}^{2}\right)$ & $21.01 \pm 2.27$ & $20.42 \pm 1.48$ & $20.72 \pm 1.02$ & 0.52 \\
\hline
\end{tabular}

Probability value $(p)$ for one way ANOVA test

Table 2. Mean thoracic, lumbar, right pelvic, left pelvic, and cervical angles of groups A, B, and C

\begin{tabular}{lcccccl}
\hline \multirow{2}{*}{ Angles } & Group A & Group B & Group C & \multicolumn{3}{c}{$p$ (Cohen’s $d$ ) } \\
\cline { 2 - 7 } & Mean $\pm S D$ & Mean $\pm S D$ & Mean $\pm S D$ & A vs. B & A vs. C & B vs. C \\
\hline Thoracic angle $\left(^{\circ}\right)$ & $47.25 \pm 6.47$ & $55.75 \pm 7.89$ & $32.18 \pm 6.77$ & $0.001(1.31)$ & $0.001(0.31)$ & $0.001(2.98)$ \\
Lumbar angle $\left(^{\circ}\right)$ & $54.75 \pm 5.38$ & $65.33 \pm 3.22$ & $32.18 \pm 6.15$ & $0.001(1.96)$ & $0.001(4.19)$ & $0.001(10.29)$ \\
Right pelvic angle $\left(^{\circ}\right)$ & $24.01 \pm 2.4$ & $24.58 \pm 2.26$ & $14.13 \pm 1.7$ & $0.66(0.23)$ & $0.001(4.11)$ & $0.001(4.62)$ \\
Left pelvic angle $\left(^{\circ}\right)$ & $24 \pm 1.77$ & $24.66 \pm 2.2$ & $14.6 \pm 1.65$ & $0.49(0.37)$ & $0.001(5.31)$ & $0.001(4.57)$ \\
Cervical angle $\left(^{\circ}\right)$ & $26.8 \pm 2.39$ & $41.87 \pm 2.54$ & $21.37 \pm 1.66$ & $0.001(6.3)$ & $0.001(2.27)$ & $0.001(8.07)$ \\
\hline
\end{tabular}

Significance level was set as $\alpha=0.05$; probability value $(p)$ for one way MANOVA test 
This finding is in line with the studies reporting that biomechanical alignment changes and musculoskeletal problems are prevalent in women with CPP $[5,6]$. With regard to the pelvic alignment changes and CPP, the current results showed that there were pelvic alignment changes with cyclic CPP. That is in agreement with a previous paper by Kim et al. [7], who investigated the link between pelvic torsion alignment and cyclic menstrual pain and found a positive relationship between them. One possible explanation is that the motions of the lumbosacral vertebrae are abnormally limited in dysmenorrhoeic women, so the body fluid within the pelvic region rises and uterus contraction contributes to the intensification of the menstrual pain. Likewise, women who exhibited a pelvis imbalance suffered more from pain [8].

A possible explanation of that is related to the instability of the pelvis: the change of the uterine position causes an imbalance in hormone levels; oxytocin and prostaglandin, which change the contractile activity of the uterus, can lead to blood flow and hormone alternation [4]. The pelvic instability might be caused by chronic inflammation in the genitourinary system or by anatomical disorders in venous return $[15,16]$. The pelvic stability reduced in patients with CPP related to disorders of the musculoskeletal system results in an abnormal motor pattern and deficiencies in postural adaptations due to increased muscle tightness and tender points causing a 'pelvic-pain-protecting pattern,' which acts as a guarded behaviour in patients with pain; this also adds more inadequate postural stability [15]. A 'pelvic-pain-protecting pattern' may itself lead to hampered venous drainage, as observed in the dilation of pelvic veins. Thus, the vascular phenomena may be considered secondary to the primary muscle dysfunction, leading to increased tissue fluid and hypoxemia of local tissue [16].

As for the cervical spine alignment changes and CPP, the current results showed a statistically significant difference in cervical alignment changes in women with CPP. That is in line with a previous paper by Montenegro et al. [6], who made a comparison between healthy female volunteers and women who suffered from CPP and observed a statistically significant difference only for the upper part of the body. Lee et al. [17] found that the cervical spine had an impact on the tone of the lumbopelvic musculature, particularly in the gluteal region. This impact was explained by Willard et al. [18], who assumed that the entire body was connected by mechanical chains, i.e. functional linking of the structural components of the axial skeleton that affects each segment by muscular, fascial, and ligamentous interconnections. The lateral side fascia connects the functional structure of the quadratus lumborum and scalene muscle by the agonist muscles of the neck side bending and the pelvic lateral tilting angle. Considering that the changes observed in women with CPP resulted from a vicious cycle of pain, antalgic postures acquired over time and postural impairments can contribute significantly to the maintenance or worsening of pain [19].

With reference to the thoracic and lumbar alignment changes and CPP, the current results showed a statically significant difference in thoracic alignment changes in women with CPP. This is in agreement with the previous papers of posture classification, which reported that a higher percentage of women with CPP exhibited increased thoracic kyphosis and lumbar lordosis [5].

One of the possible explanations of the thoracicpelvic alignment relation is that structural instability reduces spinal mobility and vascular supply, leading to cyclic pain as a result of vasoconstriction [20].

In the context of the lumbar spine changes and CPP, the current results demonstrated a statistically significant difference in the lumbar spine alignment angles in women with CPP. This is in line with Kim et al. [7], who studied the relationship between pelvic alignment and cyclic menstrual pain: they found a statistically significant difference in lumbar and thoracic alignment changes [21]. The possible physiological explanation is that the changes observed in women with cyclic CPP owing to a vicious pain cycle result from the defensive movement pattern due to the perception of pain and antalgic postures acquired over time. The postural impairments may lead to a significant reduction of blood circulation and lower lymph drainage of tissue fluids due to the reduction of diaphragm movement; this results in pelvic floor muscle tension and the lower spine rotation [21]. The possible intersegmental explanation is that since the lumbosacral vertebrae have abnormally limited movement, body fluid rises in the pelvic region and uterine contraction leads to menstrual pain intensification [7]. One explanation depends on the referred pain pattern from musculoskeletal dysfunctions in the muscles of the lumbopelvic area: this may be responsible for symptoms associated with CPP [7].

The results of this study conflict with a previous work by Montenegro et al. [6], who investigated the postural changes in women with and without CPP and found a non-significant difference in the lumbar spine and thoracic spine pelvis alignment changes between women with and without CPP. One of the 
R.M. Elbesh et al., Biomechanical changes in chronic pelvic pain

possible explanations of a significant increase in spinal alignment changes was that in the case of noncyclic $\mathrm{CPP}$, the central nervous system (CNS) was central to the experience of pain, and chronic pain conditions, in general, are associated with alterations in both the structure and function of the CNS conditions [22]. Otherwise, in the case of menstrual pain associated with alterations in the function of the CNS conditions, central processing alteration of experimental noxious stimuli, the hypothalamic-pituitary-adrenal axis dysfunction, and reduced quality of life during menstruation cause no deactivation of brain regions in response to the harmful stimulation in women who suffer from menstrual pain [22].

This study would be of valuable benefit to physical therapists and healthcare providers mainly dealing with spine problems, posture correction, and pelvis problems treatment protocols in females. It aimed to determine the biomechanical alignment changes that might occur in women with CPP to find the effect on the configuration of the spine and pelvis objectively. Also, the study compared the configuration of the spinopelvic alignments with normal women to reduce the long-term impact of CPP, which potentially affects the shape of the spine because of incorrect posture.

This study had some limitations. The hormonal profile for each participant was not measured. The participants were evaluated only on the first day after the menstrual cycle. There were no subclasses of cyclic and noncyclic CPP. Functional scales were not applied. Detailed assessments are required to achieve a better differential diagnosis and, hence, better therapy for these women, as well as to recognize the complex links between the pelvis, pain, and dysfunctions in other areas of the body.

\section{Conclusions}

The study showed that biomechanical alignment changes were more frequent in women with noncyclic or cyclic CPP.

\section{Disclosure statement}

No author has any financial interest or received any financial benefit from this research.

\section{Conflict of interest}

The authors state no conflict of interest.

\section{References}

1. Daniels JP, Khan KS. Chronic pelvic pain in women. BMJ. 2010;341:c4834; doi: 10.1136/bmj.c4834.
2. Bonnema R, McNamara M, Harsh J, Hopkins E. Primary care management of chronic pelvic pain in women. Cleve Clin J Med. 2018;85(3):215-223; doi: 10.3949/ccjm.85a.16038.

3. Won HR, Abbott J. Optimal management of chronic cyclical pelvic pain: an evidence-based and pragmatic approach. Int J Womens Health. 2010;2:263-277; doi: 10.2147/IJWH.S7991.

4. Witzeman KA, Kopfman JE. Obstetrics-gynecology resident attitudes and perceptions about chronic pelvic pain: a targeted needs assessment to aid curriculum development. J Grad Med Educ. 2014;6(1):39-43; doi: 10.4300/JGME-D-13-00053.1.

5. Mieritz RM, Thorhauge K, Forman A, Mieritz HB, Hartvigsen J, Christensen HW. Musculoskeletal dysfunctions in patients with chronic pelvic pain: a preliminary descriptive survey. J Manipulative Physiol Ther. 2016;39(9):616-622; doi: 10.1016/j.jmpt.2016.09. 003.

6. Montenegro ML, Mateus-Vasconcelos EC, Rosa e Silva JC, Dos Reis FJC, Nogueira AA, Poli-Neto OB. Postural changes in women with chronic pelvic pain: a case control study. BMCMusculoskelet Disord. 2009;10:82; doi: 10.1186/1471-2474-10-82.

7. Kim M-J, Baek I-H, Goo B-O. The effect of lumbarpelvic alignment and abdominal muscle thickness on primary dysmenorrhea. J Phys Ther Sci. 2016;28(10): 2988-2990; doi: 10.1589/jpts.28.2988.

8. Le Huec JC, Aunoble S, Philippe L, Nicolas P. Pelvic parameters: origin and significance. Eur Spine J. 2011; 20(Suppl. 5):564-571; doi: 10.1007/s00586-011-1940-1.

9. Herrington L. Assessment of the degree of pelvic tilt within a normal asymptomatic population. Man Ther. 2011;16(6):646-648; doi: 10.1016/j.math.2011.04.006.

10. McFarland C, Wang-Price S, Richard S. Clinical measurements of cervical lordosis using flexirule and inclinometer methods in individuals with and without cervical spine dysfunction: a reliability and validity study. J Back Musculoskelet Rehabil. 2015;28(2):295302; doi: 10.3233/bmr-140517.

11. Kolber MJ, Pizzini M, Robinson A, Yanez D, Hanney WJ. The reliability and concurrent validity of measurements used to quantify lumbar spine mobility: an analysis of an iPhone ${ }^{\circledR}$ application and gravity based inclinometry. Int J Sports Phys Ther. 2013;8(2):129-137.

12. Macintyre NJ, Bennett L, Bonnyman AM, Stratford PW. Optimizing reliability of digital inclinometer and flexicurve ruler measures of spine curvatures in postmenopausal women with osteoporosis of the spine: an illustration of the use of generalizability theory. ISRN Rheumatol. 2011;2011:571698; doi: 10.5402/2011/57 1698.

13. Clarkson HM. Musculoskeletal assessment: joint motion and muscle testing. Wolters Kluwer/Lippincott Williams \& Wilkins Health; 2013.

14. Bruno AG, Anderson DE, D’Agostino J, Bouxsein ML. The effect of thoracic kyphosis and sagittal plane align- 
ment on vertebral compressive loading. J Bone Miner Res. 2012;27(10):2144-2151; doi: 10.1002/jbmr.1658.

15. Grover S, Srivastava A, Lee R, Tewari AK, Te AE. Role of inflammation in bladder function and interstitial cystitis. Ther Adv Urol. 2011;3(1):19-33; doi: 10.1177/ 1756287211398255.

16. Durham JD, Machan L. Pelvic congestion syndrome. Semin Intervent Radiol. 2013;30(4):372-380; doi: 10.1055/s-0033-1359731.

17. Lee HS, Chung HK, Park SW. Correlation between trunk posture and neck reposition sense among subjects with forward head neck postures. Biomed Res Int. 2015;2015:689610; doi: 10.1155/2015/689610.

18. Willard FH, Vleeming A, Schuenke MD, Danneels L, Schleip R. The thoracolumbar fascia: anatomy, function and clinical considerations. J Anat. 2012;221(6): 507-536; doi: 10.1111/j.1469-7580.2012.01511.x.

19. Origoni M, Leone Roberti Maggiore U, Salvatore S, Candiani M. Neurobiological mechanisms of pelvic pain. Biomed Res Int. 2014;2014:903848; doi: 10.1155/2014/ 903848.

20. Sedighimehr N, Manshadi FD, Shokouhi N, Baghban AA. Pelvic musculoskeletal dysfunctions in women with and without chronic pelvic pain. J Bodyw Mov Ther. 2018;22(1):92-96; doi: 10.1016/j.jbmt.2017.05.001.

21. Brawn J, Morotti M, Zondervan KT, Becker CM, Vincent K. Central changes associated with chronic pelvic pain and endometriosis. Hum Reprod Update. 2014; 20(5):737-747; doi: 10.1093/humupd/dmu025.

22. Vincent K, Warnaby C, Stagg CJ, Moore J, Kennedy S, Tracey I. Dysmenorrhoea is associated with central changes in otherwise healthywomen. Pain. 2011;152(9): 1966-1975; doi: 10.1016/j.pain.2011.03.029. 